\title{
Influência do combustível (diesel e biodiesel) e das características da frota de veículos do transporte coletivo de Curitiba, Paraná, nas emissões de NOx
}

\author{
Influence of fuel (diesel and biodiesel) features and fleet vehicles \\ transport group of NOx emissions in Curitiba, Paraná, Brazil \\ Wellington Ferreira Ribas', Patrícia Bilotta², Paulo Roberto Janissek ${ }^{3}$, \\ Marco Aurélio da Silva Carvalho Filho ${ }^{4}$, Renato Arruda Penteado Neto ${ }^{5}$
}

\begin{abstract}
RESUMO
O sistema de transporte coletivo da cidade de Curitiba, no Paraná, é reconhecido pelas suas soluções inovadoras e modelo para o Brasil. Neste trabalho foi analisada a influência do combustível (diesel e biodiesel) e das condições da frota de veículos do transporte coletivo de Curitiba nas emissões de NOx, e essa é uma iniciativa pioneira. Foram selecionados 188 veículos (9,7\% do total) com motores de 7 a 12 L, denominados B7R, B1OM, B12M e B215RH, e foram realizadas medições de $\mathrm{NO}$ e $\mathrm{NO}_{2}$ na saída dos escapamentos. Com relação ao tipo de combustível, as emissões de NO e $\mathrm{NO}_{2}$ para o biodiesel (B100) foram, respectivamente, 37 e $26 \%$ superiores aos valores observados com o combustível diesel (S1O). Comparando motores B12M com até 3 e 10 anos de operação, as emissões médias de NOx foram próximas de $40 \%$ maior. Assim, foi possível observar que os veículos a biodiesel tiveram maior emissão de NOx quando comparados com veículos a diesel, em condições similares de operação e tempo de uso do motor. Entretanto, embora o uso de biodiesel traga vantagens ambientais, como energia renovável, novas tecnologias e dispositivos devem ser desenvolvidos para controlar emissões de $\mathrm{NO}$ e $\mathrm{NO}_{2}$ em motores que utilizam esse tipo de combustível. Para trabalhos futuros, os autores recomendam testes em dinamômetro, para simular emissões de NOx em motores a diesel e biodiesel em diferentes condições de carga e regime de rotação do motor.
\end{abstract} Palavras-chave: biodiesel; diesel; NOx; transporte coletivo; Curitiba.

\begin{abstract}
The public transportation system in Curitiba, Paraná, Brazil, is recognized by its innovative solutions; and is a model for Brazil. In this work, the influence of fuel (diesel and biodiesel) and the conditions of the public transport vehicle fleet of Curitiba in NOx emissions was assessed, and this is a pioneered initiative. A total of 188 vehicles ( $9.7 \%$ of the total) with engines of 7 and $12 \mathrm{~L}$ were selected, named B7R, B10M, B12M and $\mathrm{B} 215 \mathrm{RH}$, and measurements of $\mathrm{NO}$ and $\mathrm{NO}_{2}$ were performed in the exhaust output. In relation to the type of fuel, the emissions of NO and $\mathrm{NO}_{2}$ for biodiesel (B100) were, respectively, 37 and $26 \%$ superior to the values observed with the fuel diesel (S10). Comparing the engines B12M with up to 3 and 10 years of operation, the average emissions of NOx were close to $40 \%$ higher. Thus, it was possible to observe that the biodiesel vehicles had higher NOx emissions when compared with diesel vehicles in similar conditions of operation and engine wear time. Therefore, although the use of biodiesel brings environmental advantages, as renewable fuel, new technologies and devices must be developed to control emissions of $\mathrm{NO}$ and $\mathrm{NO}_{2}$ in engines that use this type of fuel. For future works the authors recommend tests on dynamometer to simulate NOx emission in diesel and biodiesel engine in different conditions of charge and engine speed.
\end{abstract}

Keywords: biodiesel; diesel; NOx; public transportation; Curitiba

Trabalho realizado no Programa de Pós-graduação em Gestão Ambiental da Universidade Positivo (UP) - Curitiba (PR), Brasil. 'Mestre em Gestão Ambiental pela UP - Curitiba (PR), Brasil.

2Doutora em Engenharia Civil na Hidráulica e Saneamento pela Universidade de São Paulo (USP). Especialista em Mudanças Climáticas, Projetos Sustentáveis e Mercado de Carbono pela Universidade Federal do Paraná (UFPR). Professora Titular do Programa de Mestrado e Doutorado em Gestão Ambiental da UP - Curitiba (PR), Brasil. ${ }^{3}$ Doutor em Química Orgânica pela USP. Professor do Instituto Federal de Educação, Ciência e Tecnologia do Rio Grande do Sul (IFRS) - Caxias do Sul (RS), Brasil. 4Doutor em Química pela Universidade Estadual Paulista “Júlio de Mesquita Filho" (UNESP). Professor Titular do Programa de Mestrado e Doutorado em Gestão Ambiental da UP - Curitiba (PR), Brasil.

${ }^{5}$ Doutor em Engenharia e Ciências dos Materiais pela UFPR. Pesquisador no Instituto de Tecnologia para o Desenvolvimento (LACTEC) - Curitiba (PR), Brasil. Endereço para correspondência: Patrícia Bilotta - Rua Professor Pedro Viriato Parigot de Souza, 5.300 - Campo Comprido - 81280-330 - Curitiba (PR), Brasil - E-mail: pb.bilotta@gmail.com Recebido: 23/04/14 - Aceito: 03/11/15 - Reg. ABES: 133868 


\section{INTRODUÇÃO}

Em Curitiba, Paraná, a preocupação com a qualidade do meio ambiente surgiu já nos anos 1940, com a elaboração do Plano Agache, um dos primeiros planos diretores urbanos implementados no país. Nesse documento havia propostas para o saneamento básico, o descongestionamento de vias urbanas e a estruturação de setores especiais de habitação, comércio e indústria (KUCZKOWSKI; KUCZKOWSKI; GRECO-FERLIZI, 2014).

A história do transporte começa em 1887 com a "Empreza Curitybana" com o primeiro bonde puxado por animais. No início do século XX (1903), o número de passageiros era da ordem de $680 \mathrm{mil}$, passando para a segunda metade da década seguinte para a quantidade de 1,9 milhão de passageiros ao ano. Com a concessão dada a 13 empresas, em 1955 a cidade contava com uma frota de 50 ônibus e 80 lotações. Com o crescimento da cidade, em 1965 foi editado o Plano Diretor de Transportes de Curitiba, criando vias estruturais como eixo de movimentação urbana. Em 1970, com uma frota de 673 veículos, transportava-se um total de 515 mil passageiros por dia. Em setembro de 1974, o então prefeito Jaime Lerner inaugurou a primeira linha de ônibus expresso, chamado na época de "Vermelhão". Esse sistema transportava aproximadamente 1,9 milhão de passageiros por mês (KUCZKOWSKI; KUCZKOWSKI; GRECO-FERLIZI, 2014).

A partir da década de 1980, os terminais de ônibus passam a ser fechados e os usuários passaram a utilizar catracas de acesso. Com essa nova sistemática foi possível implantar a tarifa única. Os usuários podiam trocar de linha dentro dos terminais sem pagar nova passagem. Com esse novo sistema, consolidou-se então a Rede Integrada de Transporte (RIT). Em 1980 começaram a circular os ônibus articulados em substituição aos tradicionais "Vermelhões".

No início da década de 1990, a Volvo do Brasil começou a desenvolver o primeiro ônibus biarticulado brasileiro. Era um veículo com 25 metros de comprimento e com capacidade de transporte de 270 passageiros. Nesse mesmo período foram implantadas as Linhas Diretas popularmente chamadas de "Ligeirinhos". O Sistema Integrado de Transporte Coletivo (SITC) atualmente transporta 2,2 milhões de passageiros diariamente e a frota é composta por 1930 ônibus, que atendem 395 linhas (KUCZKOWSKI; KUCZKOWSKI; GRECO-FERLIZI, 2014).

Com o aumento da população em áreas urbanas e a necessidade de constantes deslocamentos, houve um aumento no consumo de combustíveis derivados do petróleo. A queima desses combustíveis gera uma série de poluentes para o meio ambiente, como o monóxido de carbono, óxidos de nitrogênio e gases de efeito estufa. Tem-se estudado combustíveis alternativos com o intuito de reduzir a concentração destes na atmosfera. Combustíveis tais como o etanol e o biodiesel são fontes renováveis de energia, considerados menos poluentes.

Em 2 de julho de 2003 a presidência da república instituiu, por meio de decreto, um grupo de trabalho interministerial para avaliar a viabilidade do uso de biodiesel na matriz energética brasileira. A implantação do Programa Nacional de Produção e Uso do Biodiesel (PNPB) foi estabelecida por meio de decreto em 23 de dezembro de 2003 (BRASIL, 2003).

Curitiba mostra com orgulho o título de capital ecológica e tem um sistema de transporte coletivo que é considerado modelo. O sistema atende, além da capital, outros 12 municípios integrados ao Sistema de Transporte de Curitiba. Com uma frota crescente que transporta diariamente cerca de 2.225.000 passageiros, uma pequena parte dessa frota utiliza esse combustível alternativo (KUCZKOWSKI; KUCZKOWSKI; GRECO-FERLIZI, 2014). Porém, aumentar o número de veículos da frota de transporte coletivo com o uso de biodiesel ou veículos híbridos (veículos com motorização elétrica e combustão interna) pode acarretar aumento na produção de NOx.

O aumento do número de veículos em circulação somado à deficiência apresentada nos sistemas de transporte coletivo acarreta um tráfego intenso nos grandes centros urbanos. O excesso de veículos causa um aumento na poluição atmosférica em níveis superiores ao que a natureza pode absorver. Esse crescimento afeta diretamente o custo nas áreas de saúde pública e infraestrutura, isto é, resulta na necessidade de implementação de infraestruturas para locomoção (vias urbanas, rodovias, viadutos, etc.) e estruturas ligadas diretamente à saúde da população (hospitais e centros de saúde).

O transporte coletivo urbano é basicamente movido pela queima de combustíveis fósseis (diesel) e renováveis (biodiesel). A queima de combustíveis renováveis e não renováveis é um dos responsáveis pela emissão de poluentes nocivos à saúde e que degradam o ambiente urbano. Dentre esses poluentes, pode-se destacar: o monóxido de carbono (CO), os hidrocarbonetos (HC), os materiais particulados (MP), os óxidos de nitrogênio (NOx) e os óxidos de enxofre (SOx) (CARVALHO, 2011).

Existem diversos padrões de emissões veiculares no mundo. A esses padrões são estabelecidos limites para emissão de NOx. Em 1991 surgiram na Europa normas de emissão de gases tóxicos liberada pelo escapamento de veículos automotores. Essas normas foram denominadas "Euro" e têm como objetivo o controle da poluição emitida por veículos automotores (EURO-LEX, 2014). As legislações sobre emissões veiculares relacionam os seguintes poluentes: NOx, MP, CO e HC. No Brasil, a regulamentação é preconizada pelo Programa de Controle da Poluição do Ar por Veículos Automotores (PROCONVE). Em 2012 entrou em vigor a fase P-7 do PROCONVE que define limites de emissões para veículos pesados do ciclo Diesel. Essa fase equivale ao Euro V (CONAMA, 2008).

O objetivo deste trabalho foi verificar a influência do combustível (diesel e biodiesel) e das condições da frota de veículos do transporte coletivo de Curitiba nas emissões de $\mathrm{NOx}\left(\mathrm{NO}_{\text {e }} \mathrm{NO}_{2}\right)$, visando ampliar o conhecimento sobre essa questão.

\section{Estado da arte}

Os restritos limites de emissões de material particulado e de NOx impostos aos fabricantes de motores do ciclo Diesel têm estimulado o desenvolvimento de soluções para o pós-tratamento de gases emitidos por esse 
tipo de motor. Atualmente, a redução do NOx ocorre por meio de um processo de redução catalítica (SCR), com adição de ureia, ou pela recirculação dos gases do motor (EGR), ou ainda pela associação de ambas em um conjunto de filtros (FENG \& LU, 2015). Os modernos sistemas de pós-tratamento de gases de exaustão de motores a Diesel contêm um catalisador de oxidação (DOC), um filtro de material particulado de alta eficiência (DPF), um sistema SCR e um catalisador (AMOX), com a finalidade de minimizar os efeitos negativos da presença da amônia para o meio ambiente (KHALEK et al., 2015). O sistema SCR é uma forma efetiva de redução do NOx, porém em regime transiente, onde estão presentes rápidas mudanças nas cargas e nas temperaturas de exaustão, o controle do sistema ainda se configura como um grande desafio operacional (FENG \& LU, 2015). Para se obter reduções expressivas no uso do SCR é necessária a utilização de catalisadores de alta eficiência na conversão de NOx (CASANOVA et al., 2015).

Rakopoulos, Rakopoulos e Giakoumis (2015) investigaram as emissões de NOx por motores alimentados com uma mistura de diesel e biodiesel (a partir de etanol, n-butanol e óleo de algodão) em diferentes proporções. $\mathrm{O}$ estudo mostrou que o aumento na parcela de biodiesel na mistura modifica a relação ar-combustível na câmara de combustão, favorecendo a formação de NOx. Segundo Li et al. (2015), os fatores que exercem influência na emissão de NOx em motores que operam com uma mistura de diesel e biodiesel são: o tipo de motor, o regime de rotação (constante ou variado) e a carga efetiva do motor (até $8 \%$ em função da carga). Xue, Grift e Hansen (2011) constataram que não há um consenso em relação aos resultados obtidos pela substituição (parcial ou total) de biodiesel por diesel convencional, porém $65 \%$ dos 69 artigos revisados reportaram um aumento das emissões de NOx quando biodiesel era utilizado em substituição ao diesel convencional. Fazal, Haseeb e Masjuki (2011) também realizaram uma ampla revisão dos artigos publicados a respeito da utilização do biodiesel, indicando uma tendência à redução das emissões quando álcoois são misturados ao diesel, e de aumento das emissões de NOx quando óleos vegetais são incorporados ao diesel convencional.

O biodiesel é obtido a partir de ácidos graxos presentes em óleos vegetais (LIMA et al., 2007), microalgas (AHMAD et al., 2011), de óleos alternativos, como o babaçu (LIMA et al., 2007) e a semente de girassol (ANTOLIN et al., 2002) e até mesmo de resíduos de óleo de cozimento (PREDOJEVIC, 2008). Algumas alternativas têm sido consideradas para ampliar o potencial energético do biodiesel, principalmente o processo de transesterificação com álcoois de pequena cadeia (PREDOJEVIC, 2008).

\section{Formação de NOx}

McCormick et al. (2001), em seus estudos, buscam inferir primeiramente sobre os mecanismos de formação de NOx nos motores de combustão, destacando três processos de formação:

NOx térmico: em temperaturas elevadas, tais como aquelas que ocorrem no interior da câmara de combustão de um motor a diesel,
$\mathrm{N}_{2}$ e $\mathrm{O}_{2}$ podem reagir por meio de reações em cadeia conhecidas como mecanismo Zeldovich. As Equações 1 a 3 representam o mecanismo de Zeldovich para a geração de NOx por reações que envolvem o nitrogênio atmosférico e ocorrem durante a combustão;

$$
\begin{array}{ll}
\mathrm{O}+\mathrm{N}_{2} \rightleftharpoons \mathrm{NO}+\mathrm{N} & \Delta \mathrm{H}=+75 \mathrm{kcal} . \mathrm{mol}^{-1} \quad\left(\mathrm{~T}>1500^{\circ} \mathrm{C}\right) \\
\mathrm{N}+\mathrm{O}_{2} \rightleftharpoons \mathrm{NO}+\mathrm{O} & \Delta \mathrm{H}=-32 \mathrm{kcal} \cdot \mathrm{mol}^{-1} \\
\mathrm{~N}+\mathrm{OH} \rightleftharpoons \mathrm{NO}+\mathrm{H} & \Delta \mathrm{H}=-47 \mathrm{kcal} \cdot \mathrm{mol}^{-1}
\end{array}
$$

- Prompt de NOx: a formação de "prompt NOx" (também conhecido como "Fenimore NOx") envolve fragmentos de hidrocarbonetos intermediários da combustão de combustíveis - em particular $\mathrm{CH}$ e $\mathrm{CH}_{2}$ - que reagem com $\mathrm{N}_{2}$ na câmara de combustão;

- NOx combustível: espécies contendo nitrogênio são oxidadas a NO durante o processo de combustão dentro de um motor a diesel.

A geração de NOx pode variar dependendo de vários fatores, como tipo de motor e configuração, ciclo de serviço de injeção de combustível, controle de emissões (MCCORMICK et al., 2001). A alta temperatura de combustão faz com que ocorra a dissociação das moléculas de oxigênio em átomos. Logo após, atacam as moléculas de nitrogênio no ambiente para a formação de NO. A taxa de conversão é proporcional à concentração de átomos de oxigênio (MCCORMICK et al., 2001).

$\mathrm{O}$ uso de biodiesel pode resultar em benefícios, pela diminuição das emissões de CO, HC e MP. O aumento de NOx é tratado como um problema que deve ser atenuado. A diminuição desses problemas pode ser feita a partir de modificações no motor e/ou alterações no combustível. Paralelamente, deve-se proceder o controle dos gases e partículas provenientes da combustão (HOEKMAN \& ROBBINS, 2012).

\section{Alternativas para a redução de emissão de NOx em motores a biodiesel}

O sistema de recirculação de gases (EGR) consiste em outra técnica comumente usada para a redução de emissões de NOx em motores a diesel e biodiesel. Essa técnica consiste no reaproveitamento dos gases de escape para serem admitidos na fase de admissão. A recirculação dos gases de escape diminui a temperatura local na câmara de combustão. Para uma eficiente reação de oxidação é necessário excesso de oxigênio, uma mistura de gases ( $\mathrm{NO}, \mathrm{O}_{2}, \mathrm{NH}_{3}$ ) e temperatura entre 700 e $800^{\circ} \mathrm{C}$ (TESFA et al., 2012).

Outra forma de controlar emissões de gases é a redução catalítica seletiva (Selective Catalityc Reduction - SCR). Nesse sistema é utilizado um agente redutor líquido automotivo (ARLA 32). O ARLA 32 é um reagente líquido produzido à base de ureia (32\%), que tem o uso específico para aplicação veicular. O agente redutor é pulverizado no gás de escapamento, ocorrendo uma reação química no catalisador que praticamente neutraliza a geração de NOx. O Ibama determina a obrigatoriedade do uso do ARLA 32, a partir de 2012, em veículos novos que utilizam diesel como combustível (CONAMA, 2009). 


\section{METODOLOGIA}

A metodologia utilizada neste trabalho foi dividida em duas etapas, sendo:

1. medição de $\mathrm{NO}$ e $\mathrm{NO}_{2}$ e estimativa de $\mathrm{NOx}$ no escapamento de veículos do transporte coletivo de Curitiba movidos a biodiesel e diesel;

2. análise e comparação da emissão de $\mathrm{NO}, \mathrm{NO}_{2}$ e $\mathrm{NOx}$ entre os veículos da frota de Curitiba para os combustíveis biodiesel e diesel.

\section{Etapa I: medição de $\mathrm{NO}$ e $\mathrm{NO}_{2}$}

Nessa etapa foram feitos contatos junto às empresas de transporte coletivo de Curitiba e também junto à Urbanização de Curitiba S.A. (URBS). O objetivo das visitas foi determinar o tamanho e a característica da frota de ônibus. Segundo a operadora do SITC a frota de ônibus apresenta um total de 1.930 veículos de modelos e capacidades de passageiros diversas.

Os modelos escolhidos para este estudo foram os veículos que apresentam motor Volvo e utilizam combustível óleo diesel (S10/B5) ou biodiesel (B100). Os veículos que utilizam biodiesel são em número de 29. Foram feitas análises em 20 veículos da categoria Ligeirão que apresentaram um consumo de $1.060 \mathrm{~m}^{3}$ de biodiesel para percorrer 1.319.150 $\mathrm{km}$ no ano de 2013. Foram medidos também 150 veículos que utilizam como combustível óleo diesel S10. Esses 150 veículos percorreram em 2013 um total de $9.010 .790 \mathrm{~km}$ e consumiram $5.345 \mathrm{~m}^{3}$ de combustível. Atualmente fazem parte da frota do transporte coletivo de Curitiba 79 veículos híbridos. Desses, foram medidos 18 veículos que percorreram, em 2013, 1.118.284 km com um gasto de $470 \mathrm{~m}^{3}$ de óleo diesel.

A coleta de gases de exaustão foi feita diretamente na saída do duto de descarga dos veículos submetidos às medições. Para realizar essas medições foi utilizado o equipamento Tempest 100. A Tabela 1 apresenta as principais especificações técnicas do aparelho Tempest 100 para os parâmetros de interesse. O equipamento mede os gases de interesse $\left(\mathrm{NO}, \mathrm{NO}_{2}\right)$ e indica os valores obtidos em um visor. Para a estimativa da emissão de NOx foi considerada a somatória da média de emissão de $\mathrm{NO}$ e $\mathrm{NO}_{2}$, pois são os principais contribuintes nas emissões de NOx por veículos a combustão.

Foram realizadas medições em 188 veículos. Para essas medições os veículos encontravam-se parados e o motor funcionando em marcha lenta na garagem das empresas de transporte coletivo. Todos os veículos medidos estavam em processo de retorno à garagem, e apresentavam um uso mínimo de três horas de trabalho.

Os modelos estudados foram: B7, B10, B12M e B215RH (ônibus híbrido). A Tabela 2 apresenta as especificações técnicas dos tipos de motores analisados nesta pesquisa, cujas informações foram obtidas junto às gerências de manutenção das empresas de transporte coletivo de Curitiba nas visitas realizadas para as medições dos gases $\mathrm{NO}_{\text {e }} \mathrm{NO}_{2}$.

Um modelo interessante é o B215RH, o híbrido da Volvo. O modelo apresenta um motor diesel e outro elétrico, que tracionam o veículo de forma simultânea ou independente. Na partida, o ônibus é movido pelo motor elétrico. $\mathrm{O}$ alto torque proporciona uma partida macia e silenciosa. A partir do momento em que a velocidade de $20 \mathrm{~km} \cdot \mathrm{h}^{-1}$ é atingida, o motor diesel entra em operação. Quando o veículo está parado, seja no trânsito, em paradas de embarque ou desembarque ou nos semáforos, $\mathrm{o}$ motor diesel é desligado automaticamente. $\mathrm{O}$ uso reduzido de energia e as baixas emissões são os principais benefícios desse tipo de motor.

Outro item avaliado foi a idade do veículo. Conforme critério das gerências de manutenção das empresas visitadas, são considerados veículos novos aqueles com até três anos de uso. Os veículos de 7 até 10 anos estão em fase final de vida útil. De acordo com a regulamentação da URBS, a vida útil de um ônibus é de 10 anos.

Para organizar a coleta de dados dos veículos junto às empresas foi utilizada uma planilha. A planilha apresenta os seguintes itens:

- $\quad$ identificação: caracteriza o número do veículo da frota;

- $\quad \mathrm{km}$ : número de quilômetros registrados no odômetro no dia da coleta;

- cor: as diversas categorias das linhas de transporte são identificadas pela cor do ônibus;

- motor: motorização do veículo;

- $\quad$ ano: ano de fabricação.

Após a coleta de dados foi feito um cruzamento entre as informações obtidas considerando-se três variáveis: combustível, modelo do veículo e idade do veículo. O cruzamento de dados permitiu a comparação em duas situações distintas:

1. comparação da emissão de gases por faixa de idade do veículo com motores equivalentes e o combustível utilizado (diesel e biodiesel);

2. comparação da emissão de gases por faixa de idade para um mesmo combustível e motores diferentes.

Tabela 1 - Especificação técnica do aparelho Tempest 100 para os parâmetros de interesse.

\begin{tabular}{|c|c|c|c|}
\hline Parâmetro medido & Faixa & Resolução & Precisão \\
\hline \multirow{2}{*}{$\begin{array}{l}\text { Óxido de } \\
\text { nitrogênio (NO) }\end{array}$} & \multirow{2}{*}{ 0-1.000 ppm } & \multirow{2}{*}{$1 \mathrm{ppm}$} & $<100$ ppm: \pm 5 ppm \\
\hline & & & $>100$ ppm: $\pm 5 \%$ \\
\hline \multirow{2}{*}{$\begin{array}{l}\text { Dióxido de } \\
\text { nitrogênio }\left(\mathrm{NO}_{2}\right)\end{array}$} & \multirow{2}{*}{ 0-200 ppm } & \multirow{2}{*}{$1 \mathrm{ppm}$} & $<20$ ppm: \pm 5 ppm \\
\hline & & & $>20$ ppm: $\pm 5 \%$ \\
\hline
\end{tabular}

Fonte: White Gas (2015)

Tabela 2 - Especificações técnicas dos motores analisados nesta pesquisa.

\begin{tabular}{l|c|c|c|c} 
Especificações & B7M & B10M & B12M & B215RH \\
\hline Cilindros & 6 & 2 & 6 & 4 \\
Potência máxima & $\begin{array}{c}213 \mathrm{~kW}(290 \\
\mathrm{HP}) \\
2300 \mathrm{rpm}\end{array}$ & $\begin{array}{c}266 \mathrm{~kW} \\
(360 \mathrm{HP}) \\
2500 \mathrm{rpm}\end{array}$ & $\begin{array}{c}250 \mathrm{~kW} \\
(340 \mathrm{HP}) \\
1800 \mathrm{rpm}\end{array}$ & $\begin{array}{c}161 \mathrm{~kW}(215 \\
\mathrm{HP})\end{array}$ \\
$\begin{array}{l}2300 \mathrm{rpm} \\
\text { Torque máximo }\end{array}$ & $\begin{array}{c}1200 \mathrm{Nm} \\
1200-1700 \mathrm{rpm}\end{array}$ & $\begin{array}{c}1500 \mathrm{Nm} \\
1200 \mathrm{rpm}\end{array}$ & $\begin{array}{c}1700 \mathrm{Nm} \\
1200 \mathrm{rpm}\end{array}$ & $\begin{array}{c}816 \mathrm{Nm} \\
1200-1700 \\
\mathrm{rpm}\end{array}$ \\
\hline Cilindrada & $\begin{array}{c}7,14 \mathrm{dm}^{3} \\
(7,14 \mathrm{~L})\end{array}$ & $\begin{array}{c}9,6 \mathrm{dm}^{3} \\
(9,6 \mathrm{~L})\end{array}$ & $\begin{array}{c}12,13 \mathrm{dm}^{3} \\
(12,13 \mathrm{~L})\end{array}$ & $\begin{array}{c}1,19 \mathrm{dm}^{3} \\
(1,19 \mathrm{~L})\end{array}$ \\
\hline
\end{tabular}




\section{Etapa II: análise e comparação da emissão de $\mathrm{NO}, \mathrm{NO}_{2}$ e $\mathrm{NOx}$}

Os dados foram agrupados de acordo com os seguintes critérios:

1. tipo de combustível: biodiesel e diesel;

2. tipo de motor do veículo: B7R, B10, B12M e B215RH;

3. idade do veículo (ano de fabricação do veículo);

4. total de quilômetros percorridos em 2013;

5. quilômetros do odômetro;

6. total de litros de combustível gasto em 2013. Para cada emissão medida ( $\mathrm{NO}, \mathrm{NO}_{2}$ e $\mathrm{NOx}$ ) foram determinados os valores médio, máximo e mínimo, considerando-se as 188 coletas realizadas.

\section{RESULTADOS E DISCUSSÃO}

A composição da frota do transporte coletivo de Curitiba é bem diversificada (Tabela 3). A operadora do sistema, URBS, define a categoria da linha e o tipo de veículo a ser utilizado. A categoria Ligeirão é constituída apenas por veículos movidos a biodiesel. Na categoria Expresso, dos 133 biarticulados apenas 2 utilizam biodiesel. O uso de motores Volvo é distribuído nas demais linhas, sendo complementadas por motores Mercedes Bens,
Scania e Volkswagen. Destacam-se na composição da frota os veículos híbridos modelo B215RH, que apresentam motores a combustão e elétrico.

\section{Comparação da emissão de NO e NOx por faixa de idade do veículo com motores equivalentes e o combustível utilizado (diesel e biodiesel)}

A comparação de combustíveis diferentes para motores equivalentes (B12M) foi analisada na faixa de tempo de uso de 0 a 3 anos. Observou-se que ocorreu um aumento da emissão de NO quando o veículo utiliza como combustível B100. Quando comparados veículos com o mesmo tempo de uso, o aumento é de $37,14 \%$. A emissão de $\mathrm{NO}_{2}$ para veículos nas mesmas condições de uso é, em média, 4,2 vezes maior quando o combustível é o S10 e da ordem de 32,8 $\pm 1,6 \mathrm{ppm}$. A Tabela 4 permite observar que ocorre uma emissão média maior quando utilizado o biodiesel em relação ao diesel.

Portanto, com base nos testes realizados, o principal contribuinte para a formação de NOx é a emissão de NO. As emissões de NOx provenientes da combustão de B100 são aproximadamente $26 \%$ maiores do que as emissões resultantes da utilização de S10. Observou-se uma emissão média de NOx de 432,3 ppm para veículos movidos a B100, enquanto a média para veículos movidos a 10 foi de 342,4 ppm.

Tabela 3 - Caracterização da frota de veículos do transporte coletivo de Curitiba.

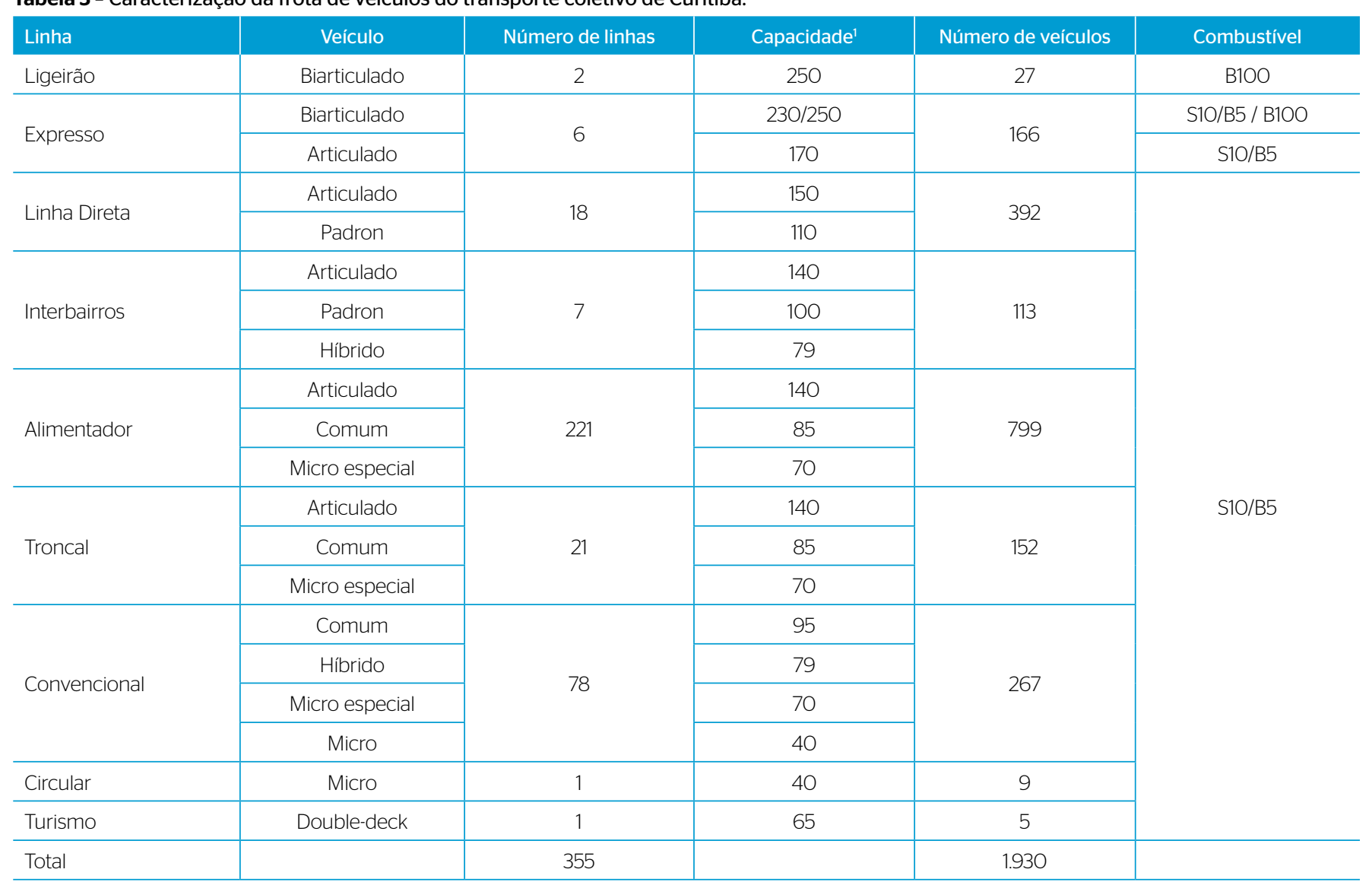

'Número máximo de passageiros no veículo. Fonte: URBS (2013). 
A figura 1 mostra uma interpretação comparativa dos dados apresentados na Tabela 4, considerando a emissão de $\mathrm{NO}, \mathrm{NO}_{2}$ nos veículos movidos a S10 (diesel) e B100 (biodiesel).

Conforme mostra a Figura 1, as emissões de NO para veículos com o mesmo tempo de uso ( 0 a 3 anos) que utilizaram B100 foram, em média, 424,6 ppm, enquanto os veículos que utilizaram S10 emitiram 309,6 ppm. Observa-se que as emissões foram 1,4 vezes maiores nos veículos que utilizam B100. Já as emissões de $\mathrm{NO}_{2}$ medidas por veículos que utilizaram $\mathrm{B} 100$ apresentaram valores de 7,8 ppm (em média) contra a média de 32,8 ppm por veículos movidos a S10. As emissões de NOx por veículos que utilizaram B100 foram de 432,3 ppm, com um valor máximo de 496,0 ppm e um mínimo de 369,0 ppm. Com uma emissão máxima de 437,0 ppm, os veículos que utilizam $S 10$ apresentaram uma média de 342,4 ppm para um mínimo de 315,0 ppm. As emissões totais de $\mathrm{NO}_{\text {e } \mathrm{NO}_{2}}$ (NOx) foram $26 \%$ maiores para os veículos movidos a biodiesel para a mesma vida útil de trabalho.

Tabela 4 - Emissão de NO, $\mathrm{NO}_{2}$ e NOx para emissões provenientes do uso de biodiesel e diesel.

\begin{tabular}{|c|c|c|c|c|c|}
\hline \multicolumn{3}{|c|}{ Biodiesel } & \multicolumn{3}{c|}{ Diesel } \\
\hline $\begin{array}{c}\text { O a 3 } \\
\text { anos }\end{array}$ & $\begin{array}{c}\text { 3 a 7 } \\
\text { anos }\end{array}$ & $\begin{array}{c}7 \text { a 10 } \\
\text { anos }\end{array}$ & $\begin{array}{c}\text { 0 a 3 } \\
\text { anos }\end{array}$ & $\begin{array}{c}\text { 3 a 7 } \\
\text { anos }\end{array}$ & $\begin{array}{c}7 \text { a 10 } \\
\text { anos }\end{array}$ \\
\hline
\end{tabular}

$\mathrm{NO}(\mathrm{ppm})$

\begin{tabular}{c|c|c|c|c|c|c}
\hline Máximo & 488,0 & - & - & 395,0 & 320,0 & - \\
\hline Mínimo & 364,0 & - & - & 285,0 & 293,0 & - \\
\hline Média & 424,6 & - & - & 309,6 & 308,4 & - \\
\hline $\mathrm{NO}_{2}(\mathrm{pppm})$ & \multicolumn{7}{|l}{} \\
\hline Máximo & 12,0 & - & - & 42,0 & 33,8 & - \\
\hline Mínimo & 5,0 & - & - & 30,0 & 30,9 & - \\
\hline Média & 7,8 & - & - & 32,8 & 32,6 & - \\
\hline NOx (ppm) & \multicolumn{7}{|l}{} \\
\hline Máximo & 496,0 & - & - & 437,0 & 353,8 & - \\
\hline Mínimo & 369,0 & - & - & 315,0 & 323,9 & - \\
\hline Média & 432,3 & - & - & 342,4 & 341,0 & - \\
\hline
\end{tabular}

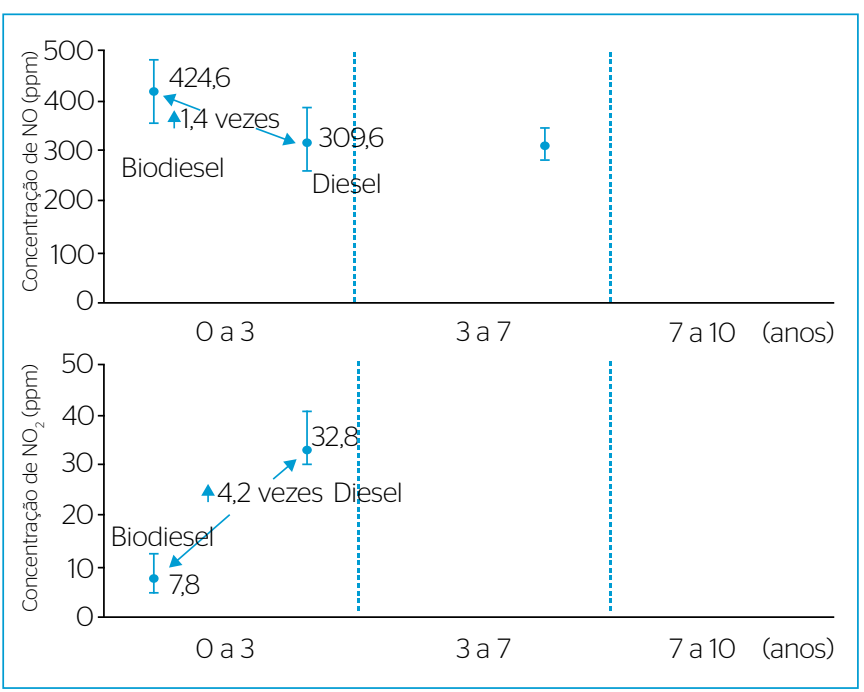

Figura 1 - Emissão de $\mathrm{NO}$ e $\mathrm{NO}_{2}$ para biodiesel e diesel.

\section{Comparação da emissão de $\mathrm{NO}, \mathrm{NO}_{2}$ e $\mathrm{NO}$ por faixa de idade para um mesmo combustível e motores diferentes}

A tabela 5 mostra a variação das concentrações de $\mathrm{NO}, \mathrm{NO}_{2}$ e $\mathrm{NOx}$. Foram considerados veículos com motorizações diferentes para um único tipo de combustível (S10).

Tabela 5 - Resultado da medição das concentrações de $\mathrm{NO}, \mathrm{NO}_{2}$ e cálculo de NOx para os veículos movidos a diesel em diferentes configuraçỗes de motor.

\begin{tabular}{|c|c|c|c|c|c|}
\hline & & & Máximo & $\begin{array}{c}\text { Mínimo } \\
\text { ppm }\end{array}$ & Média \\
\hline \multirow{6}{*}{ B7R } & \multirow{3}{*}{ Ano 2008} & NO & 347 & 335 & 340,3 \\
\hline & & $\mathrm{NO} 2$ & 36,6 & 35,4 & 35,9 \\
\hline & & NOx & 383,6 & 370,4 & 376,3 \\
\hline & \multirow{3}{*}{ Ano 2011} & NO & 305 & 285 & 294,9 \\
\hline & & $\mathrm{NO} 2$ & 32,2 & 30 & 31,1 \\
\hline & & NOx & 337,2 & 315,1 & 326 \\
\hline \multirow{6}{*}{$\mathrm{B} 1 \mathrm{O}$} & \multirow{3}{*}{ Ano 2000} & NO & 531 & 528 & 529,5 \\
\hline & & $\mathrm{NO} 2$ & 56,1 & 55,8 & 55,9 \\
\hline & & NOx & 587,1 & 583,8 & 585,4 \\
\hline & \multirow{3}{*}{ Ano 2003} & NO & 445 & 442 & 443,5 \\
\hline & & $\mathrm{NO} 2$ & 47 & 46,7 & 46,8 \\
\hline & & NOx & 492 & 488,7 & 490,3 \\
\hline \multirow{27}{*}{$\mathrm{B} 12 \mathrm{M}$} & \multirow{3}{*}{ Ano 2001} & $\mathrm{NO}$ & 515 & 502 & 508,5 \\
\hline & & $\mathrm{NO} 2$ & 54 & 53 & 53,5 \\
\hline & & NOx & 569 & 555 & 562 \\
\hline & \multirow{3}{*}{ Ano 2003} & NO & 455 & 452 & 453,5 \\
\hline & & $\mathrm{NO} 2$ & 48 & 48 & 48 \\
\hline & & NOx & 503 & 500 & 501,5 \\
\hline & \multirow{3}{*}{ Ano 2004} & NO & 441 & 441 & 441 \\
\hline & & $\mathrm{NO} 2$ & 46,6 & 46,6 & 46,6 \\
\hline & & NOx & 487,6 & 487,6 & 487,6 \\
\hline & \multirow{3}{*}{ Ano 2006} & $\mathrm{NO}$ & 399 & 375 & 386 \\
\hline & & $\mathrm{NO} 2$ & 42,1 & 39,6 & 40,8 \\
\hline & & NOx & 441,1 & 414,6 & 426,8 \\
\hline & \multirow{3}{*}{ Ano 2008} & $\mathrm{NO}$ & 362 & 301 & 346,3 \\
\hline & & $\mathrm{NO} 2$ & 38 & 31,8 & 36,6 \\
\hline & & $\mathrm{NOX}$ & 400 & 332,8 & 382,9 \\
\hline & \multirow{3}{*}{ Ano 2009} & $\mathrm{NO}$ & 344 & 290 & 312,3 \\
\hline & & $\mathrm{NO} 2$ & 36 & 30,6 & 32,9 \\
\hline & & $\mathrm{NOX}$ & 380 & 320,6 & 345,2 \\
\hline & \multirow{3}{*}{ Ano 2010} & $\mathrm{NO}$ & 320 & 293 & 308,4 \\
\hline & & $\mathrm{NO} 2$ & 33,8 & 30,9 & 32,6 \\
\hline & & NOx & 353,8 & 323,9 & 341 \\
\hline & \multirow{3}{*}{ Ano 2011* } & $\mathrm{NO}$ & 395 & 285 & 309,6 \\
\hline & & $\mathrm{NO} 2$ & 42 & 30 & 32,8 \\
\hline & & NOx & 437 & 315 & 342,4 \\
\hline & \multirow{3}{*}{ Ano $2011^{\star *}$} & $\mathrm{NO}$ & 488 & 364 & 424,6 \\
\hline & & $\mathrm{NO} 2$ & 12 & 5 & 7,8 \\
\hline & & NOx & 496 & 369 & 432,3 \\
\hline \multirow{3}{*}{$\mathrm{B} 215 \mathrm{RH}$} & \multirow{3}{*}{ Ano 2012} & $\mathrm{NO}$ & 370 & 348 & 361,2 \\
\hline & & $\mathrm{NO} 2$ & 42 & 35 & 38,1 \\
\hline & & NOX & 410 & 386 & 399,2 \\
\hline
\end{tabular}

B7R: motor 290 HP; B1OM: motor 360 HP; B12M: motor 340 HP; B215RH: motor 215 HP (veículo híbrido); *veículo articulado; **veículo biarticulado. 
Para um mesmo combustível (S10) foi observado que, para veículos de mesma potência, um maior tempo de uso acarreta maior emissão de $\mathrm{NO}$ e $\mathrm{NO}_{2}$. Um veículo na faixa de uso de 7 a 10 anos apresentou emissões 1,7 vezes maior do que um veículo com até 3 anos de uso, para motores de diferentes potências. As emissões medidas para os motores B12M com até 3 anos de uso foram, em média, $40 \%$ menores do que um motor equivalente com 10 anos de uso. Os veículos com motores B7R com até 3 anos de uso emitiram, em média, 31,1 ppm de $\mathrm{NO}_{2}$. Já os veículos com tempo de uso entre 3 e 7 anos emitiram 35,9 ppm desse mesmo gás. Observou-se também que os veículos equipados com motores B12M apresentaram um aumento de 39,6\% para as emissões de $\mathrm{NO}_{2}$. Veículos com tempo de uso de 0 a 3 anos emitiram em média 32,8 ppm e os veículos com tempo de uso de 7 a 10 anos emitiram 45,8 ppm (em média).

A emissão de NOx também aumenta com o tempo de uso dos veículos. Foi encontrada uma emissão mínima (315,0 ppm) de NOx para veículos de modelo B12M (0 a 3 anos) e uma emissão máxima de 587,1ppm para o modelo B10M com tempo de uso de 7 a 10 anos. Conclui-se que a emissão de NOx também aumenta em função do tempo de uso do veículo. A tabela 6 apresenta os valores encontrados nas medições dos gases $\mathrm{NO}, \mathrm{NO}_{2}$ e $\mathrm{NOx}$ (soma de $\mathrm{NO}$ e $\mathrm{NO}_{2}$ ) levando em consideração os modelos dos veículos e o intervalo do tempo de uso dos veículos.

Pode-se observar na figura 2 que os valores médios de emissões de NO são 22,5\% menores para o modelo B7R. Esses modelos emitem em média 294,9 ppm de NO contra 361,2 ppm do modelo B215RH para o mesmo tempo de uso ( 0 a 3 anos). Quando comparados os modelos B10M e B12M com tempo de uso superior a 7 anos, observa-se que os modelos B10M emitem 12\% mais NO do que o modelo B12M.

Quanto às emissões de NO medidas em relação aos modelos B7R e B215R para veículos na faixa entre 0 e 3 anos, o modelo B215RH emitiu 22,5\% a mais de NO do que o modelo B7R. A figura também apresenta as emissões médias de NO medidas em relação aos modelos B10M e B12M. Os veículos com tempo de uso entre 7 e 10 anos, equipados com motores B10M, emitiram $12,0 \%$ a mais de NO do que os veículos dotados de motores B12M. Para os veículos B215RH observou-se $22,5 \%$ mais emissões de NO do que nos veículos equipados com motores B7M para a mesma faixa de tempo de uso do veículo. As emissões provenientes dos motores B12M foram 309,6 ppm e ficaram um pouco acima dos modelos $\mathrm{B} 7 \mathrm{M}$, que apresentaram valores médios de 294,9 ppm.

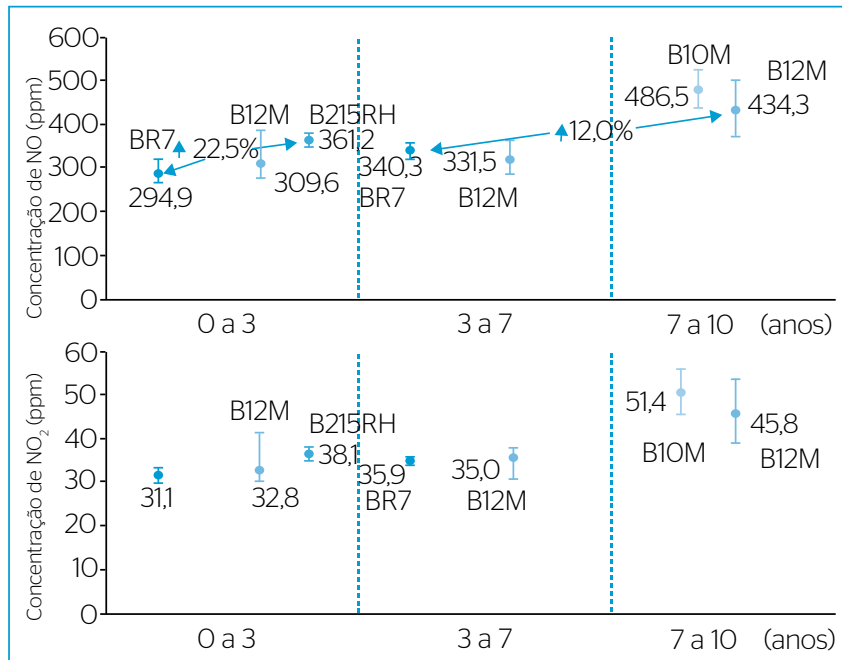

B7R: motor 290 HP; B1OM: motor 360 HP; B12M: motor 340 HP; B215RH: motor 215 HP (veículo híbrido).

Figura 2 - Comparativo de emissões de $\mathrm{NO}$ e $\mathrm{NO}_{2}$ para diferentes tipos de motores (B7R, B10M, B12M e B215RH) com o uso de um mesmo combustível (diesel).

Tabela 6 - Emissões provenientes de diferentes tipos de motores com o uso de um mesmo combustível (S10).

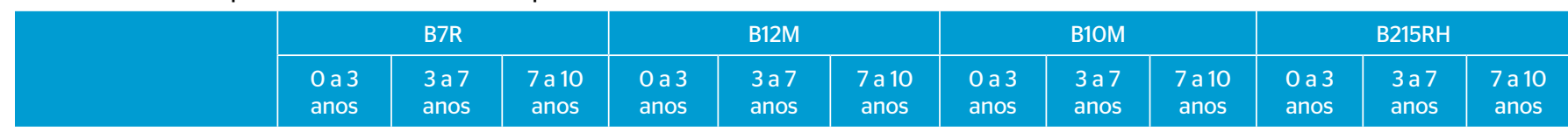

\begin{tabular}{|c|c|c|c|c|c|c|c|c|c|c|c|c|}
\hline \multicolumn{13}{|l|}{$\mathrm{NO}$ (ppm) } \\
\hline Máximo & 305,0 & 347,0 & - & 395,0 & 362,0 & 515,0 & - & - & 531,0 & 370,0 & - & - \\
\hline Mínimo & 285,0 & 335,0 & - & 285,0 & 290,0 & 375,0 & - & - & 442,0 & 348,0 & - & - \\
\hline Média & 294,9 & 340,3 & - & 309,6 & 331,5 & 434,3 & - & - & 486,5 & 361,2 & - & - \\
\hline \multicolumn{13}{|l|}{$\mathrm{NO}_{2}(\mathrm{ppm})$} \\
\hline Máximo & 32,2 & 36,6 & - & 42,0 & 38,0 & 54,0 & - & - & 56,1 & 42,0 & - & - \\
\hline Mínimo & 30,0 & 35,4 & - & 30,0 & 30,6 & 39,6 & - & - & 46,7 & 35,0 & - & - \\
\hline Média & 31,1 & 35,9 & - & 32,8 & 35,0 & 45,8 & - & - & 51,4 & 38,1 & - & - \\
\hline \multicolumn{13}{|l|}{ NOx (ppm) } \\
\hline Máximo & 337,2 & 383,6 & - & 437,0 & 400,0 & 441,1 & - & - & 587,1 & 410,0 & - & - \\
\hline Mínimo & 315,1 & 370,4 & - & 315,0 & 320,6 & 414,6 & - & - & 488,7 & 386,0 & - & - \\
\hline Média & 326,0 & 376,3 & - & 342,4 & 366,5 & 426,8 & - & - & 537,9 & 399,2 & - & - \\
\hline
\end{tabular}

B7R: motor 290 HP; B1OM: motor 360 HP; B12M: motor 340 HP; B215RH: motor 215 HP (veículo híbrido). 
A quantidade de $\mathrm{NO}_{2}$ emitida por veículos B215RH foi 22,5\% maior do que nos veículos com motores B7R quando considerada a mesma faixa de tempo de vida dos veículos. O uso de combustível S10 em veículos com mais de 7 anos de vida apresentou um aumento das emissões de $\mathrm{NO}_{2}$ de 12,0\% para os motores B10M em relação aos motores B12M. Observa-se também que as emissões de $\mathrm{NO}_{2}$ dos veículos com faixa intermediária de uso (7 a 10 anos) ficaram muito próximas (variação de 2,5\%) quando comparados os motores B7R e B12M. Os veículos com tempo de uso de 0 a 3 anos apresentaram uma variação de $22,5 \%$ nas emissões de $\mathrm{NO}_{2}$. Pode-se observar que o veículo híbrido (B215RH), nas condições do estudo apresentou, uma emissão média igual a 38,1 ppm de $\mathrm{NO}_{2}$ e o modelo $\mathrm{B} 7 \mathrm{R}$ com a menor média teve uma emissão de 31,1 ppm.

\section{CONCLUSÃO}

Dos 188 veículos investigados, com o mesmo tipo de motor (B12M), observou-se que os veículos movidos a biodiesel B100 apresentaram emissão em média 26\% maior de NOx do que aqueles que usam diesel S10. Veículos movidos a diesel apresentaram emissões de NO menores do que veículos que utilizaram biodiesel. Veículos movidos a biodiesel apresentaram emissões de $\mathrm{NO}$ superiores às emissões de $\mathrm{NO}_{2}$. A menor emissão de NOx foi encontrada nos veículos B12M fabricados em 2011. Os veículos B10M fabricados em 2000 apresentaram as maiores emissões de NOx. Com o uso de biodiesel os veículos com motores B12M apresentaram emissões 37\% maiores do que os veículos de mesma motorização que utilizaram óleo diesel.

Os veículos híbridos (B215RH) apresentaram emissões médias de 399,2 ppm de NOx. Esses valores são 22,5\% maiores do que o observado nos veículos com motores $B 7 R$, que emitiram em média 326,0 ppm de NOx para o mesmo intervalo de tempo de uso (0 a 3 anos).

Os resultados indicam o aumento das emissões de $\mathrm{NO}_{2}$ e principalmente NO com a utilização de motores a biodiesel. Assim, para que as vantagens ambientais desse combustível renovável possam ser maximizadas, novas tecnologias/dispositivos para o controle de emissões, sobretudo de NO, devem ser considerados.

\section{REFERÊNCIAS}

AHMAD, A.L.; YASIN, N.H.M.; DEREK, C.J.C.; LIM, J.K. (2011) Microalgae as a sustainable energy source for biodiesel production: a review. Renewable and Sustainable Energy Reviews, v. 15, n. 1, p. 584-593.

ANTOLIN, G.; TINAUT, F.V.; BRICEÑO, Y.; CASTAÑO, V.; PÉREZ, C.; RAMIREZ, A.I. (2002) Optimisation of biodiesel production by sunflower oil transesterification. Bioresource Technology, v. 83, n. 2, p. 111-114.

BRASIL. (2003) Decreto de 23 de dezembro de 2003. Institui a Comissão Executiva Interministerial encarregada da implantação das ações direcionadas à produção e ao uso de óleo vegetal biodiesel como fonte alternativa de energia. Disponível em: http:// www.planalto.gov.br/ccivil_03/dnn/2003/Dnn10093.htm. Acesso em: 15 dez. 2013.

CARVALHO, C.H.R. (2011) Emissões relativas de poluentes do transporte motorizado de passageiros nos grandes centros urbanos brasileiros. Brasília: IPEA. 39 p.

CASANOVA, M.; NODARI, L.; SAGAR, A.; SCHERMANZ, K.; TROVARELLI, A. (2015) Preparation, characterization and NH3-SCR activity of FeVO4 supported on $\mathrm{TiO}_{2}-\mathrm{WO}_{3}-\mathrm{SiO}$. Applied Catalysis B: Environmental, v. 176-177, p. 699-708.

CONAMA - CONSELHO NACIONAL DO MEIO AMBIENTE. (2008) Resolução CONAMA no 403, de 11 de novembro de 2008. Brasília: Diário Oficial da União; 2008. Disponível em: http://www.mma.gov.br/ port/conama/legiabre.cfm?codlegi=591. Acesso em: 28 set. 2013.

CONAMA - CONSELHO NACIONAL DO MEIO AMBIENTE. (2009) Resolução CONAMA no 414, de 24 de setembro de 2009. Brasília: Diário Oficial da União; 2009. Disponível em: http://
www.mma.gov.br/port/conama/processos/1448F242/Relat ProconveAnual_47aCTCQA_5a7jul11.pdf. Acesso em: 28 set. 2013.

EUR-LEX. (2009) European Union Law. Regulamento (CE) n. 715/2007 do Parlamento Europeu. Norma EURO V. Disponível em: http://eur-lex.europa. eu/legal-content/PT/ALL/?uri=CELEX:32007RO715. Acesso em: 25 mar. 2014

FAZAL, M.A.; HASEEB, A.S.M.A.; MASJUKI, H.H. (2O11) Biodiesel feasibility study: an evaluation of material compatibility; performance; emission and engine durability. Renewable and Sustainable Energy Reviews, v. 15, n. 2, p. 1314-1324.

FENG, T \& LÜ, L. (2015) The characteristics of ammonia storage and the development of model-based control for diesel engine urea-SCR system. Journal of Industrial and Engineering Chemistry, v. 28, p. 97-109.

KUCZKOWSKI, A.C.; KUCZKOWSKI, F.R.; GRECO-FERLIZI, N. (2014) O passeio turístico da cidade de Curitiba. Curitiba: Mikito Artes Gráficas e Editora. $140 \mathrm{p}$

HOEKMAN S.K. \& ROBBINS C. (2012) Review of the effects of biodiese on NOx emissions. Fuel Processing Technology, v. 96, p. 237-249.

KHALEK, I.A.; BLANKS, M.G.; MERRITT, P.M.; ZIELINSKA, B. (2015) Regulated and unregulated emissions from modern 2010 emissionscompliant heavy-duty on-highway diesel engines. Journal of the Air \& Waste Management Association, v. 65, n. 8, p. 987-1001.

LI, L.; WANG, J.; WANG, Z.; XIAO, J. (2015) Combustion and emission characteristics of diesel engine fueled with diesel/biodiesel/pentanol fuel blends. Fuel, v. 156, p. 211-218. 
LIMA, J.R.O.; SILVA, R.B.; SILVA, C.C.M.; SANTOS, L.S.S.; SANTOS JUNIOR., J.R.; MOURA, E.M.M.; MOURA, C.V.R. (2007) Biodiesel de Babaçu (Orbignya Sp.) obtido por via etanólica. Química Nova, v. 30, n. 3, p. 600-603.

MCCORMICK, R.L.; GRABOSKI, M.S.; ALLEMAN, T.L.; HERRING, A.M. (2001) Impact of biodiesel source material and chemical structure on emissions of criteria pollutants from a heavy-duty engine. Environmental Science and Technology, v. 35, n. 9, p. 1742-1747.

PREDOJEVIC, Z.J. (2008) The production of biodiesel from waste frying oils: a comparison of diferent purification steps. Fuel, v. 87, p. 3522-3528.

RAKOPOULOS, D.C.; RAKOPOULOS, C.D.; GIAKOUMIS, E.G. (2O15) Impact of properties of vegetable oil, bio-diesel, ethanol and n-butanol on the combustion and emissions of turbocharged HDDI diesel engine operating under steady and transient conditions. Fuel, v. 156, p. 1-19.
TESFA, B.; MISHRA, R.; GU, F.; BALL, A.D. (2012) Water injection effects on the performance and emission characteristics of a $\mathrm{Cl}$ engine operating with biodiesel. Renewable Energy, v. 37, n. 1, p. 333-344.

URBS - URBANIZAÇÃO DE CURITIBA S.A. (2013). Disponível em: http://www.urbs.curitiba.pr.gov.br/institucional/urbs-em-numeros. Acesso em: 15 fev. 2013.

XUE, J.; GRIFT, T.E.; HANSEN, A.C. (2011) Effect of biodiesel on engine performances and emissions. Renewable and Sustainable Energy Reviews, v. 15, n. 2, p. 1098-1116.

WHITE GAS. Analisador de gases de combustão e de emissões de poluente, Tempest 100. Disponível em: http://www.whitegas.com. br/analisadores-de-gas-e-combustao-tempest-100.html. Acesso em: 08 ago. 2015 